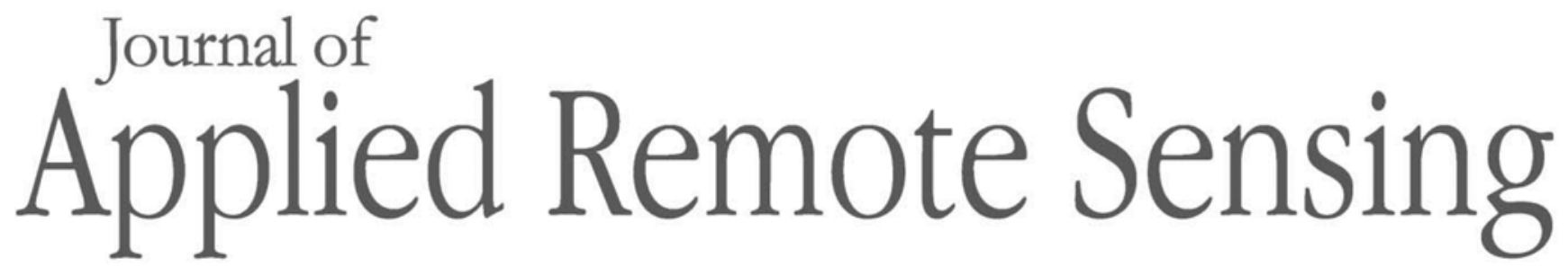

RemoteSensing.SPIEDigitalLibrary.org

\title{
Using remotely sensed spectral reflectance to indicate leaf photosynthetic efficiency derived from active fluorescence measurements
}

\author{
Yi Peng \\ Aoli Zeng \\ Tinge Zhu \\ Shenghui Fang \\ Yan Gong \\ Yanqi Tao \\ Ying Zhou \\ Kan Liu
}

Yi Peng, Aoli Zeng, Tinge Zhu, Shenghui Fang, Yan Gong, Yanqi Tao, Ying Zhou, Kan Liu, "Using remotely sensed spectral reflectance to indicate leaf photosynthetic efficiency derived from active 


\title{
Using remotely sensed spectral reflectance to indicate leaf photosynthetic efficiency derived from active fluorescence measurements
}

\author{
Yi Peng, ${ }^{a}$ Aoli Zeng, ${ }^{\text {a }}$ Tinge Zhu, ${ }^{\text {a,b }}$ Shenghui Fang, ${ }^{a}$ Yan Gong, ${ }^{a}$ \\ Yanqi Tao, ${ }^{a}$ Ying Zhou, ${ }^{\text {a }}$ and Kan Liu ${ }^{\mathrm{c}, *}$ \\ ${ }^{a}$ Wuhan University, School of Remote Sensing and Information Engineering, Lab for Remote \\ Sensing of Crop Phenotyping, Wuhan, Hubei, China \\ ${ }^{\mathrm{b}}$ Southwest Research Institute of Electronics Equipment, China Electronics Technology Group \\ Corporation, Chengdu, Sichuan, China \\ ${ }^{\mathrm{c} C h i n e s e}$ Academy of Sciences, Wuhan Institute of Physics and Mathematics, Wuhan, \\ Hubei, China
}

\begin{abstract}
Chlorophyll fluorescence (ChlF) is an important signature of photosynthesis to evaluate plant response to the environment. We explored an approach to estimate an important leaf ChlF-derived parameter, the intrinsic efficiency of photosystem II photochemistry $\left(F_{v} / F_{m}\right)$, using spectral indices calculated from leaf reflectance measured by a hyperspectral radiometer. It is observed that leaf chlorophyll content closely related to $F_{v} / F_{m}$ in nonstressed leaves, thus the indices developed for chlorophyll estimation were successfully used to estimate $F_{v} / F_{m}$. For leaves under short-term stress, $F_{v} / F_{m}$ dropped dramatically while leaf chlorophyll content remained almost the same. Compared to leaf chlorophyll content, reflectance was more sensitive to $F_{v} / F_{m}$ variations. As $F_{v} / F_{m}$ decreased, the slope of reflectance in the spectrum range of 700 to $900 \mathrm{~nm}$ obviously increased, and the first derivative reflectance in the red edge and infrared (NIR) regions was highly correlated with $F_{v} / F_{m}$. The indices using longwave red edge and NIR reflectance $\left(\mathrm{NDRE}_{740}\right.$ and $\mathrm{CI}_{740}$ ) worked well for $F_{v} / F_{m}$ retrieval in both stressed and nonstressed leaves with the coefficients of determination $\left(R^{2}\right)$ above 0.72 and normalized root-meansquare errors below 0.16 . Note that the relationships $\mathrm{NDRE}_{740}$ and $\mathrm{CI}_{740}$ versus $F_{v} / F_{m}$ were significantly different between nonstressed and stressed leaves, which may give a good implication to detect short-term stress occurrence. (c) The Authors. Published by SPIE under a Creative Commons Attribution 3.0 Unported License. Distribution or reproduction of this work in whole or in part requires full attribution of the original publication, including its DOI. [DOI: 10.1117/1.JRS.11.026034]
\end{abstract}

Keywords: chlorophyll fluorescence; chlorophyll content; photosynthesis; vegetation indices; short-term stress.

Paper 160998 received Jan. 4, 2017; accepted for publication Jun. 2, 2017; published online Jun. $19,2017$.

\section{Introduction}

Chlorophyll fluorescence (ChlF) is the red and far-red light emitted by chlorophyll pigments upon excitation with natural or artificial light in the visible and infrared (NIR) part of the spectrum. ${ }^{1}$ It is widely realized that ChlF carries valuable information for the organization, functioning, and physiology of plant photosynthesis. ${ }^{2,3}$ Energy absorbed by chlorophyll of photosystem II (PSII) is used to split water into hydrogen and oxygen, but some can be dissipated as heat or re-emitted at longer wavelengths as ChlF. The capture of ChlF variations helps understand the processes that control energy partitioning in PSII, which is useful to evaluate plant growth and stress status. ${ }^{4}$ Many studies found that ChlF is a good indicator for terrestrial gross primary production, fraction of absorbed photosynthetically active radiation (PAR), light use efficiency, and maximum carboxylation capacity. ${ }^{5-7}$ Moreover, the first

*Address all correspondence to: Kan Liu, E-mail: liuk@wipm.ac.cn 
response of a plant to environmental stress is usually an increase of nonradiative energy dissipation likely indicated by ChlF. ${ }^{8}$ Thus, ChlF usually acts as a sensitive probe for early detection of plant stress. ${ }^{9}$

The pulse amplitude-modulated (PAM) technique is widely used for leaf ChlF measurements. It employs rapid manipulations of the light regime with modulated measuring light to obtain leaf fluorescence signals under different conditions. ${ }^{4}$ The measurement is initiated with a measuring light on a dark-adapted leaf, giving the minimal level of fluorescence $\left(F_{0}\right)$ when all photochemical traps open and nonphotochemical quenching (NPQ) approaches zero. A saturating pulse of light is then applied to close nearly all photochemical traps thus fluorescence rapidly increases to a maximal level $\left(F_{m}\right)$. Turning on an actinic light can activate photosynthesis in leaf, and after a period of time, leaf fluorescence comes to a steady-state level $\left(F_{t}\right)$. By applying saturating pulses, the maximal level of fluorescence in the light $\left(F_{m}^{\prime}\right)$ can be measured. The removal of actinic light after a flash gives the minimal level of fluorescence $\left(F_{0}^{\prime}\right)$ in the light. Based on these recorded signals, various important parameters can be derived to quantify leaf photochemical and nonphotochemical processes, such as the efficiency of PSII photochemistry $\left[\phi_{\text {PSII }}=\left(F_{m}^{\prime}-F_{t}\right) / F_{m}^{\prime}\right]$, photochemical quenching $\left[\mathrm{qP}=\left(F_{m}^{\prime}-F_{t}\right) /\right.$ $\left.\left(F_{m}^{\prime}-F_{0}^{\prime}\right)\right]$, intrinsic efficiency of PSII photochemistry $\left[F_{v} / F_{m}=\left(F_{m}-F_{0}\right) / F_{m}\right]$, NPQ $\left[\mathrm{NPQ}=\left(F_{m}-F_{m}^{\prime}\right) / F_{m}^{\prime}\right]$, and so on. ${ }^{4,10}$ The development of the PAM technique has greatly facilitated the study and applications of using ChlF information to evaluate vegetation photosynthesis machinery and physiology status. Several PAM-based fluorometers have been invented to obtain ChlF information in situ, ${ }^{11}$ but they are commonly applied at leaf level and their applicability at canopy or landscape level still remains elusive., ${ }^{4,12}$

Recently, estimating ChlF from remote sensing platforms is of great interest. Unlike PAM techniques based on fluorescence measurements induced by an active light source, remote sensing approaches retrieve ChlF excited by the absorption of sunlight [i.e., solar-induced fluorescence (SIF)] using spectral reflectance. Since the fluorescence emission spectrum is superimposed on leaf or canopy reflectance that can be obtained by handheld, ground-mounted, aerial-, or space-borne sensors, remote sensing technique opens the new way for upscaling ChlF from leaf to landscape levels. For example, Moya et al. ${ }^{13}$ designed a passive instrument to detect SIF at close range for leaves in lab and for outdoor plants; Rascher et al. ${ }^{14}$ mapped the relative distribution of SIF for maize fields using spectroscope aboard aircraft; and Joiner et al. ${ }^{15}$ achieved the first observation of global terrestrial SIF using the Japanese Greenhouse Gases Observing Satellite data. Also, the European Space Agency has started the Fluorescence Explorer mission specifically for $\mathrm{ChlF}$ assessments from space. ${ }^{16,17}$ The accurate quantification of regional and global ChlF has attracted wide attention, since it is a complementary and high capacity signal for evaluating terrestrial production and global carbon cycle. ${ }^{6,18}$

Since ChlF emitted by plant under natural sunlight is very small (only $1 \%$ to $2 \%$ of the absorbed light), ${ }^{19}$ it is quite challenging to extract such weak signal from much higher detected reflectance signal. Fraunhofer line discrimination (FLD) approach was developed to retrieve SIF at the distance from close range to satellite altitudes. ${ }^{20}$ Fraunhofer lines are narrow bands at which the incident solar irradiance is strongly reduced by gaseous absorption. Several Fraunhofer lines are observed at wavelengths where the ChlF emission is relatively high (e.g., 656, 688, and $760 \mathrm{~nm}$ ), and the vegetation SIF can be retrieved based on spectral measurements inside and outside the Fraunhofer lines. ${ }^{21,22}$ FLD approach makes it viable to measure SIF from space, which is of significance for terrestrial SIF evaluation at large scale. While the PAM technique measures ChlF over quite a broad spectral region, SIF by FLD-based approach is estimated within very narrow spectral bands. So it requires the data of very high spectral resolution and large surface area to collect sufficient signal, which may be unavailable or costly for most remote sensors. ${ }^{3}$ Also, the atmospheric correction needs to be carefully conducted since gas scattering can result in large uncertainties to SIF retrivals. ${ }^{4,13}$ Moreover, to avoid reabsorption of fluorescence by chlorophyll, FLD-retrieved SIF is commonly measured at far-red bands, which may not be representative for PSII photochemistry analysis. ${ }^{4}$

In addition to the FLD-based methods, spectral vegetation indices (VIs) have been increasingly applied for remote estimation of ChlF-related parameters. They do not require reflectance exactly at the Fraunhofer lines but instead use reflectance at several bands sensitive to $\mathrm{ChlF}$ variations. These indices do not provide the direct measurements of ChlF in physical 
units but can be related to ChlF-derived information and parameters using empirical models. ${ }^{19,23,24}$

The methods using VIs are popular recently due to their simplicity and flexibility when applying to various remotely sensed data. Gitelson et al. ${ }^{25}$ showed that VIs with red edge reflectance were closely related to the ratio of leaf fluorescence at 735 to $700 \mathrm{~nm}$; Zarco-Tejada et al. ${ }^{26}$ found a good relationship between the ratio and difference VIs derived from an airborne multispectral camera and canopy SIF estimated by FLD method; and Jia et al. ${ }^{27}$ observed that red edge position of leaf reflectance is highly correlated with $F_{v} / F_{m}$ in winter wheat under different nitrogen treatments. These indices were mostly developed originally for estimating vegetation pigment-related bioparameters, such as chlorophyll content and leaf area index. They worked quite well for ChlF retrieval because ChlF in unstressed plants depends, to a great extent, on pigment content and leaf absorption. ${ }^{28-30}$ However, it has been found that conventional chlorophyll-related VIs are not efficient to capture ChlF changes in stressed vegetation. ${ }^{31}$ Indices can still remain high when plants are heavily stressed by short-term drought while fluorescence may change dramatically. ${ }^{32}$ It is observed that vegetation chlorophyll content is not directly linked to instantaneous photosynthetic processes and cannot quantify the downregulation of photosynthesis due to early environmental stresses. ${ }^{7}$ The lag for the vegetation chlorophyll to response to short-term stress can cause significant uncertainties for ChlF estimates when using empirical algorithms developed by chlorophyll-related VIs, which have not been adequately elaborated and addressed.

This study explores using vegetation reflectance to indicate variations in ChlF-derived parameters. The experiments were conducted at leaf level since it is the basic spatial scale at which fluorescence and spectral reflectance can be measured and linked simultaneously. We selected $F_{v} / F_{m}$ as the ChlF-derived parameter to be studied, since it reflects the potential quantum efficiency of PSII that usually acts as a sensitive indicator of plant photosynthesis performance. ${ }^{10}$ The $F_{v} / F_{m}$ ratio has been found rather constant in nonstressed plants. A decrease in $F_{v} / F_{m}$ can track the decrease in photochemical performance, such as sustained forms of NPQ or photoinhibition of reaction centers, when a leaf has been exposed to stress. ${ }^{33,34}$ The first objective is to test several widely used spectral VIs for $F_{v} / F_{m}$ estimation in leaves assumed under minimum stress. The second objective is to analyze the reflectance changes as leaf under short-term stress conditions and explore the indices that are closely related to $F_{v} / F_{m}$. The final objective is to explore the potential of identifying leaf stress using remotely sensed reflectance data.

\section{Materials and Methods}

Two experiments were conducted in this study. In experiment I, 48 fresh leaves were selected from aspen and cherry trees on the campus of Wuhan University, Hubei, China. These trees were planted under temperate climate with good management by campus services. In procedure of leaf selection, the spotted or crinkled leaves were avoided, and leaves appearing fresh and fully expanded were used. So, these leaves were assumed under minimum stress. The study period was from the early spring to late autumn in 2015 , and the newly sprouted, mature, and senescent leaves were all collected. The color of sample leaves varied from yellowish-green to dark green so that they could have a wide range of chlorophyll content. The in situ measurements of reflectance, chlorophyll content, and $F_{v} / F_{m}$ were conducted at leaf level. In experiment II, eight leaves were cut from trees and transported to the laboratory immediately. They were placed on a lab balcony having similar light and temperature conditions with outdoor environment but with no water supply. This experiment simulated short-term water stress at leaf level. Leaf reflectance, chlorophyll content, and $F_{v} / F_{m}$ were measured every hour after the leaf was removed from the tree until $F_{v} / F_{m}$ value was close to zero.

Reflectance spectra of the leaves were taken using a hyperspectral radiometer (ASD FieldSpec 4, ASD Inc., Boulder, Colorado) with a self-illuminated leaf clip. For each leaf, spectral reflectance in range between 400 and $900 \mathrm{~nm}$ at a spectral resolution of $3 \mathrm{~nm}$ was measured at five positions randomly distributed on the adaxial side of leaf (five scans for each position), and the median of 25 spectra scans was used as the leaf reflectance. More details can be found by Zygielbaum et al. ${ }^{35}$ 
Peng et al.: Using remotely sensed spectral reflectance to indicate leaf photosynthetic...

Table 1 Summary of VIs used in this study. $\rho_{560}, \rho_{665}, \rho_{705}, \rho_{740}, \rho_{780}, \rho_{531}$, and $\rho_{570}$ are reflectance values simulated in Sentinel-2 bands of green, red, shortwave red edge, longwave red edge, NIR regions, 531 , and $570 \mathrm{~nm}$, respectively.

\begin{tabular}{|c|c|c|}
\hline VI & Formula & Reference \\
\hline NDVI & $\mathrm{NDVI}=\left(\rho_{780}-\rho_{665}\right) /\left(\rho_{780}+\rho_{665}\right)$ & Rouse et al. ${ }^{44}$ \\
\hline SR & $\mathrm{SR}=\rho_{780} / \rho_{665}$ & Jordan $^{45}$ \\
\hline $\mathrm{EVI2}$ & $\mathrm{EVI} 2=2.5 \times\left(\rho_{780}-\rho_{665}\right) /\left(\rho_{780}+2.4 \times \rho_{665}+1\right)$ & Jiang et al. ${ }^{46}$ \\
\hline Green chlorophyll index & $\mathrm{Cl}_{\text {green }}=\left(\rho_{780} / \rho_{560}\right)-1$ & Gitelson et al. ${ }^{47,48}$ \\
\hline \multirow[t]{2}{*}{ Red edge NDVI } & $\operatorname{NDRE}_{705}=\left(\rho_{780}-\rho_{705}\right) /\left(\rho_{780}+\rho_{705}\right)$ & Gitelson and Merzlyak ${ }^{49,50}$ \\
\hline & $\operatorname{NDRE}_{740}=\left(\rho_{780}-\rho_{740}\right) /\left(\rho_{780}+\rho_{740}\right)$ & \\
\hline \multirow[t]{2}{*}{ Red edge chlorophyll index } & $\mathrm{Cl}_{705}=\left(\rho_{780} / \rho_{705}\right)-1$ & Gitelson et al. ${ }^{47,48}$ \\
\hline & $\mathrm{Cl}_{740}=\left(\rho_{780} / \rho_{740}\right)-1$ & \\
\hline MTCI & $\mathrm{MTCl}=\left(\rho_{780}-\rho_{705}\right) /\left(\rho_{705}-\rho_{665}\right)$ & Dash and Curran ${ }^{51}$ \\
\hline PRI & $\mathrm{PRI}=\left(\rho_{531}-\rho_{570}\right) /\left(\rho_{531}+\rho_{570}\right)$ & Gamon et al. ${ }^{52}$ \\
\hline
\end{tabular}

Following reflectance measurements, leaf chlorophyll content was quantified using SPAD502 (Soil and Plant Analyzer Development, Konica Minolta Inc., Ramsey, New Jersey). The SPAD can instantly provide chlorophyll meter readings based on leaf transmittance, which is widely used to assess leaf chlorophyll content. ${ }^{36-38}$ Close to positions where reflectance measurements were taken, five SPAD readings were recorded and their median was calculated as leaf chlorophyll content.

Leaf $F_{v} / F_{m}$ was measured using a PAM fluorometer (FluroPen FP 100, Photon Systems Inc., Drásov, Czech Republic). Measurements were conducted on the adaxial surface of the leaves. The leaf was first dark-adapted for 20 min using a leaf clip holder with a movable black cap. The minimum fluorescence $\left(F_{0}\right)$ and maximal fluorescence $\left(F_{m}\right)$ were then measured and recorded by FluroPen. $F_{v} / F_{m}$ was calculated as $F_{v} / F_{m}=\left(F_{m}-F_{0}\right) / F_{m}{ }^{10}$ More details about FluroPen were given by Aroca et al. ${ }^{39}$ and $\mathrm{Ni}$ et al. ${ }^{40}$

The reflectance spectra were resampled to simulate the spectral bands of Multispectral Instrument (MSI) aboard Sentinel-2. ${ }^{41}$ This satellite is showing great potential for land surface parameter estimations. ${ }^{42}$ Since the spectral response functions of the MSI spectral bands are close to rectangular, ${ }^{43}$ the simulated MSI reflectance was calculated by the average reflectance over the bandwidth of the respective MSI bands (band 1: 456 to $523 \mathrm{~nm}$, band 2: 542 to $578 \mathrm{~nm}$, band 3: 650 to $680 \mathrm{~nm}$, band 4: 697 to $713 \mathrm{~nm}$, band 5: 732 to $748 \mathrm{~nm}$, and band 6: 773 to $793 \mathrm{~nm}$ ). Using the simulated Sentinel-2 MSI reflectance, several widely used VIs were calculated (Table 1) and compared with the corresponding leaf SPAD and $F_{v} / F_{m}$ values. The sample size for this study was 48 for the experiment I and 80 for the experiment II.
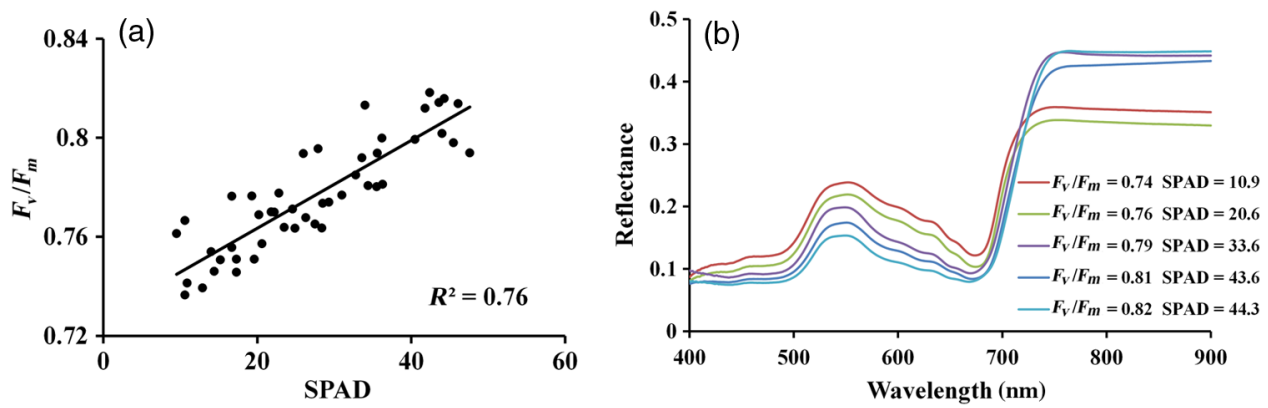

Fig. 1 (a) Relationship of relative chlorophyll content (SPAD) versus ChIF parameter $\left(F_{v} / F_{m}\right)$. (b) Spectral reflectance of nonstressed leaves at different $F_{v} / F_{m}$ and SPAD value. 


\section{Results and Discussion}

\subsection{Remote Estimation of Chlorophyll Fluorescence in Nonstressed Leaves}

In experiment I for samples assumed under minimum stress, it was observed that leaf chlorophyll varied over a wide range from SPAD values from 10 to 50, whereas $F_{v} / F_{m}$ was rather stable in the value around $0.8 \pm 8 \%$. Generally, $F_{v} / F_{m}$ positively related to SPAD with the coefficient of
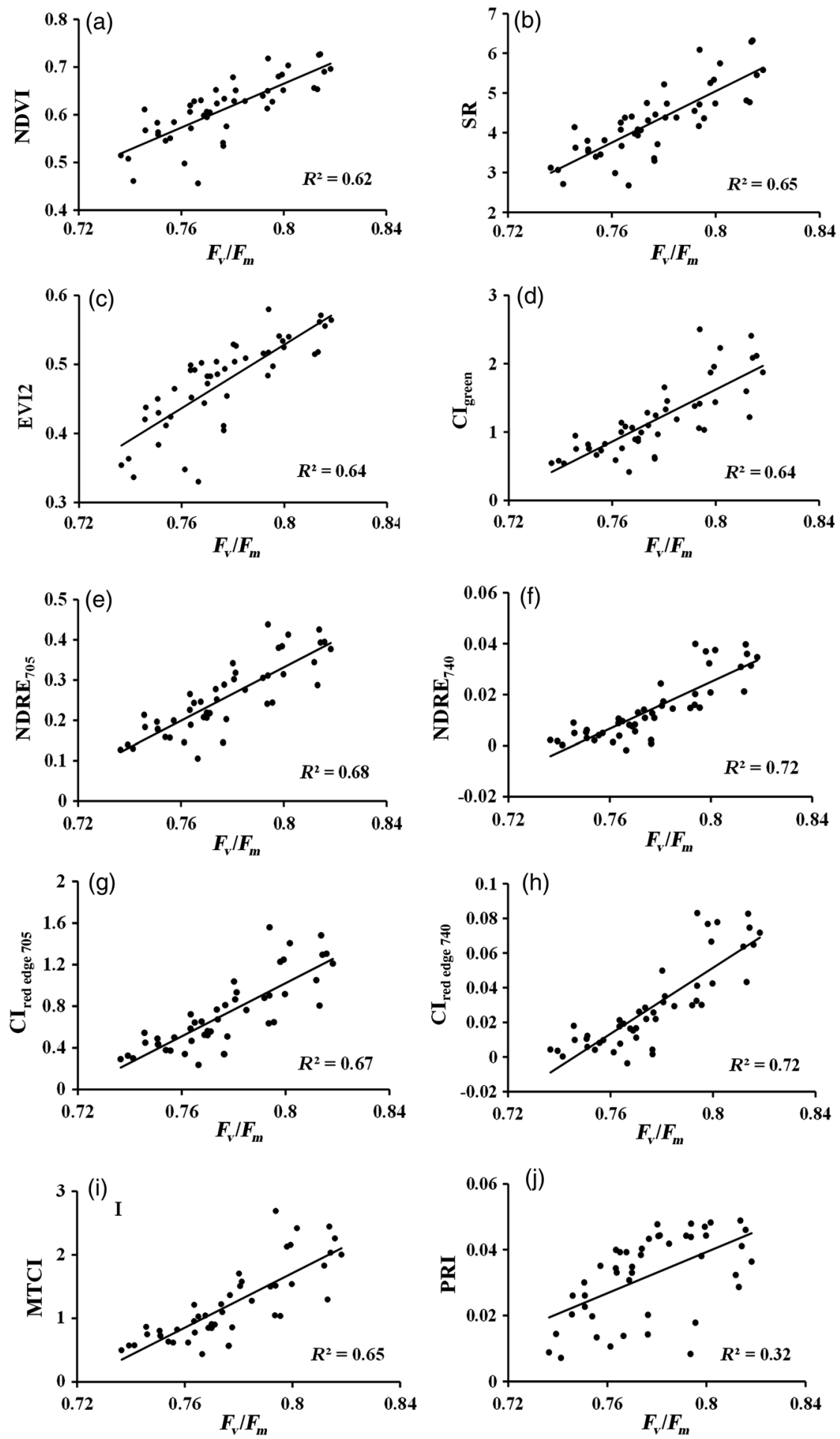

Fig. 2 Relationship between $F_{v} / F_{m}$ and (a) NDVI, (b) SR, (c) EVI2, (d) $\mathrm{Cl}_{\text {green }}$, (e) $\mathrm{NDRE}_{705}$, (f) $\mathrm{NDRE}_{740}$, (g) $\mathrm{Cl}_{705}$, (h) $\mathrm{Cl}_{740}$, (i) $\mathrm{MTCl}$, and (j) PRI in nonstressed leaves. 
Peng et al.: Using remotely sensed spectral reflectance to indicate leaf photosynthetic...

Table 2 Best-fit functions, determination coefficients $\left(R^{2}\right)$, root-mean-square errors (RMSE), and NRMSE of relationships $F_{v} / F_{m}$ versus VIs in nonstressed leaves.

\begin{tabular}{lllll}
\hline \hline VI & \multicolumn{1}{c}{ Algorithm } & $R^{2}$ & RMSE & NRMSE \\
\hline $\mathrm{NDRE}_{740}$ & $F_{v} / F_{m}=1.57 x+0.75$ & 0.72 & 0.0125 & 0.156 \\
$\mathrm{Cl}_{740}$ & $F_{v} / F_{m}=0.75 x+0.75$ & 0.72 & 0.0127 & 0.159 \\
$\mathrm{NDRE}_{705}$ & $F_{v} / F_{m}=0.21 x+0.72$ & 0.68 & 0.0129 & 0.161 \\
$\mathrm{Cl}_{705}$ & $F_{v} / F_{m}=0.05 x+0.74$ & 0.67 & 0.0128 & 0.160 \\
$\mathrm{SR}$ & $F_{v} / F_{m}=0.02 x+0.69$ & 0.65 & 0.0130 & 0.163 \\
$\mathrm{MTCl}$ & $F_{v} / F_{m}=0.03 x+0.74$ & 0.65 & 0.0131 & 0.164 \\
$\mathrm{Cl}$ & & 0.64 & 0.0134 & 0.168 \\
$\mathrm{EVI} 2$ & $F_{v} / F_{m}=0.03 x+0.74$ & 0.64 & 0.0136 & 0.170 \\
$\mathrm{NDVI}$ & $F_{v} / F_{m}=0.28 x+0.64$ & 0.62 & 0.0138 & 0.173 \\
$\mathrm{PRI}$ & $F_{v} / F_{m}=0.27 x+0.61$ & 0.32 & 0.1599 & 1.955 \\
\hline \hline
\end{tabular}

determination $\left(R^{2}\right)$ above 0.76 [Fig. 1(a)]. The measured reflectances in fresh leaves at different $F_{v} / F_{m}$ and SPAD values are presented in Fig. 1(b). With the increase in $F_{v} / F_{m}$ and SPAD, leaf reflectance decreased in PAR region (400 to $700 \mathrm{~nm}$ ) and increased in NIR region (750 to $900 \mathrm{~nm}$ ). In unstressed leaves, the increase of chlorophyll content [Fig. 1(a)] results in a slight increase in $F_{v} / F_{m}$, and leaves with higher chlorophyll content were able to absorb more PAR thus decreasing the visible reflectance. ${ }^{47}$ In addition, leaves having higher chlorophyll content were more mature and thicker, so leaf scattering became higher causing higher NIR reflectance.

Many studies used reflectance-derived indices to estimate leaf chlorophyll content accurately. ${ }^{53,54}$ Due to the close relationship between chlorophyll content and $F_{v} / F_{m}$ in unstressed leaves [Fig. 1(a)], these chlorophyll-related indices may be also useful to indicate $F_{v} / F_{m}$ variations. The relationships of $F_{v} / F_{m}$ versus VI were tested for indices that were widely applied as good proxies of leaf chlorophyll content [Figs. 2(a)-2(i)]. All chlorophyll-related VIs close to linearly related to $F_{v} / F_{m}$ with $R^{2}$ above 0.62 . Since some research suggested to use photochemical reflectance index (PRI) to indicate vegetation stress, ${ }^{55,56}$ the relationship of PRI versus $F_{v} / F_{m}$ was also tested in this study [Fig. 2(j)], but a lower correlation was found between them $\left(R^{2}=0.32\right)$.

Table 2 gives the algorithms developed for $F_{v} / F_{m}$ estimation using tested VIs. Except PRI, all indices worked well to estimate $F_{v} / F_{m}$ with normalized root-mean-square errors (NRMSE) below 0.173 (Table 2). Note that the indices using longwave red edge band (NDRE ${ }_{740}$ and $\mathrm{CI}_{740}$ ) were superior to those using shortwave red edge band, and they were the most accurate for $F_{v} / F_{m}$ retrieval among the tested indices. This is consistent with findings that the use of longwave red edge bands can give better estimates of vegetation chlorophyll content and productivity. ${ }^{43,57}$

\subsection{Remote Estimation of Chlorophyll Fluorescence in Stressed Leaves}

In experiment II for samples assumed to be under short-term stress, it is observed that in most leaves $F_{v} / F_{m}$ remained invariant during the first 4 or $5 \mathrm{~h}$ but sharply decreased afterward [e.g., Fig. 3(a)]. In experiment I, $F_{v} / F_{m}$ values of fresh leaves ranged mostly from 0.75 to 0.85 [Fig. 1(a)], which were similar with observations by Aschan et al., ${ }^{58}$ that this ratio differed insignificantly in unstressed plants. In experiment II, however, $F_{v} / F_{m}$ could drop below 0.5 after $7 \mathrm{~h}$ of leaf removal while leaf chlorophyll content varied only slightly within a day (SPAD value almost the same). $F_{v} / F_{m}$ indicates the intrinsic efficiency of PSII photochemistry, which is more sensitive to environmental stress. ${ }^{32}$ Under conditions of water stress, $F_{v} / F_{m}$ declines as the PSII reaction center is damaged in the leaf. ${ }^{59}$ However, chlorophyll content does not 

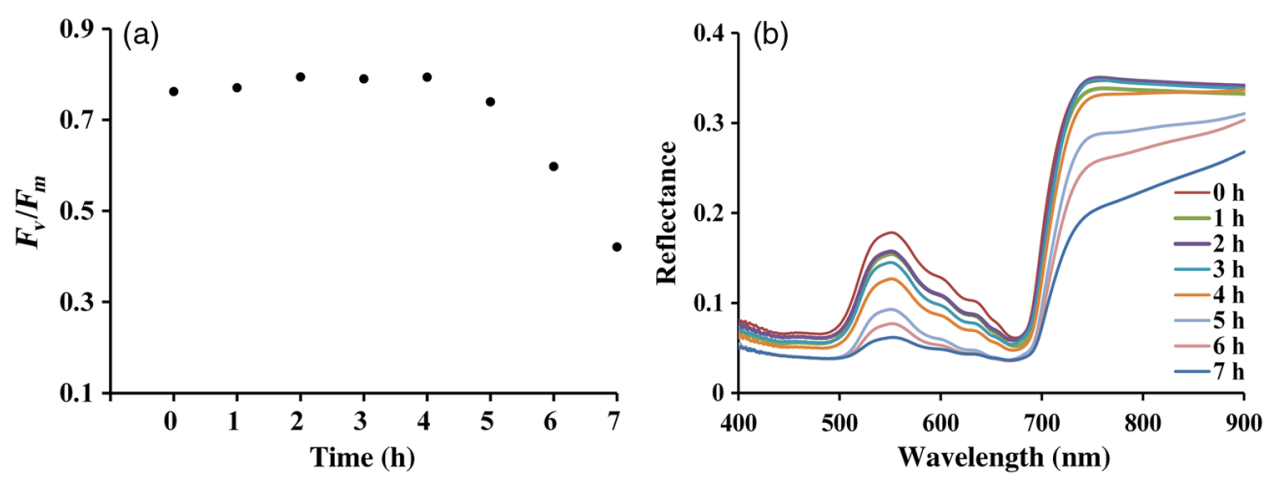

Fig. 3 (a) $F_{v} / F_{m}$ value and (b) leaf reflectance measured every hour in lab since the leaf removed from the tree with no water supply.

drop immediately in response to short-term stress. Leaf-level chlorophyll changes are only visible over time scales of days, ${ }^{60}$ because plants have evolved a number of regulatory mechanisms to modulate PAR absorption before decreasing chlorophyll pigment, ${ }^{61}$ such as leaf movements, leaf angle adjustments, ${ }^{62}$ and chloroplast avoidance movements. ${ }^{63}$ Another reason may be that the chlorophyll absorption has two reaction centers (P680 for PSII and P700 for PSI), and the dissociation of them under short-term stress may not be immediately reflected in changes of bulk chlorophyll content.

Figure 3(b) shows the leaf reflectance measured every hour since the leaf removed from the tree. More sensitive than chlorophyll content, leaf reflectance showed noticeable variations as stress became severe. The reflectance measured $5 \mathrm{~h}$ after the leaf removal was obviously different from the reflectance measured earlier, which was in agreement with $F_{v} / F_{m}$ variations shown in Fig. 3(a) $\left(F_{v} / F_{m}\right.$ dropped also after $\left.5 \mathrm{~h}\right)$. The changes of spectral reflectance for the green (530 to $560 \mathrm{~nm}$ ) and far-red to NIR (700 to $900 \mathrm{~nm}$ ) bands were the most pronounced. Before the fifth hour, reflectance reached a high and flat plateau in NIR range, and thereafter, NIR reflectance decreased but slopes in NIR and red edge regions significantly increased. In contrast, the slope in green regions slightly decreased. This is consistent with the statements by Zarco-Tejada et al. ${ }^{19,64}$ that the derivative reflectance (slope of reflectance) may enable the detection of subtle changes of $\mathrm{ChlF}$ emissions. We therefore analyzed the sensitivity of reflectance slope to $F_{v} / F_{m}$ variations at different wavelengths. Since chlorophyll exhibits fluorescence emission mainly in the red and NIR regions with peaks at $\sim 690$ and $740 \mathrm{~nm},{ }^{27}$ the first derivative of reflectance $\left(\rho^{\prime}\right)$ was calculated, and the coefficients of determination $\left(R^{2}\right)$ between $F_{v} / F_{m}$ and $\rho^{\prime}$ were plotted with wavelength in range of 600 to $900 \mathrm{~nm}$ (Fig. 4). The $R^{2}$ value peaked around $675 \mathrm{~nm}$ (above 0.8), and it remained high (between 0.80 and 0.87 ) throughout the wide spectral region of 760 to $860 \mathrm{~nm}$.

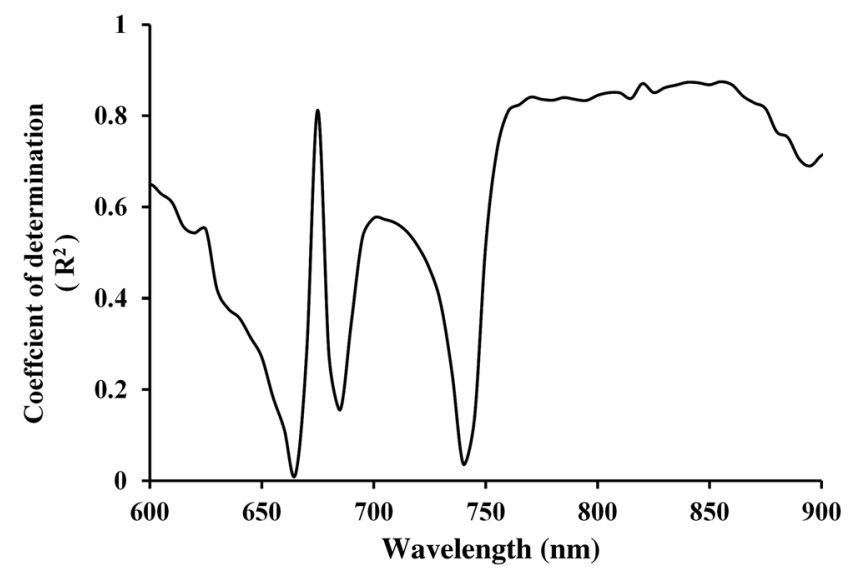

Fig. 4 The coefficient of determination $\left(R^{2}\right)$ between $F_{v} / F_{m}$ and the first derivative reflectance $\left(\rho^{\prime}\right)$ plotted with wavelength in range of 600 to $900 \mathrm{~nm}$. 

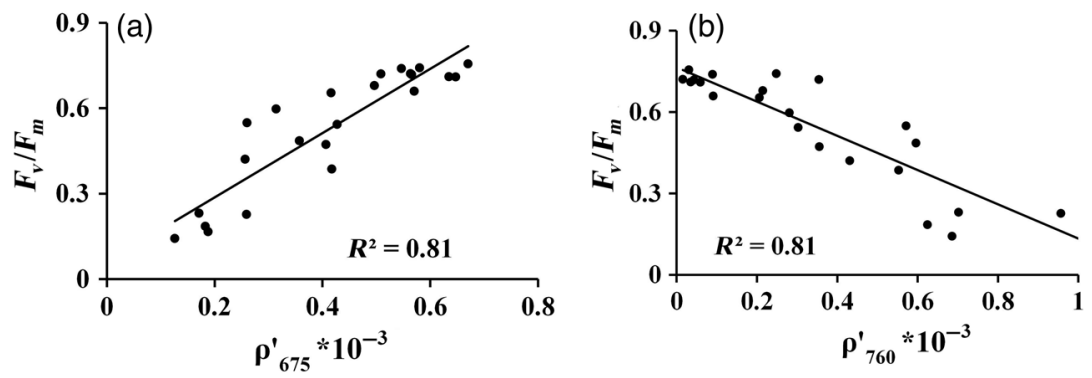

Fig. 5 Relationship between $F_{v} / F_{m}$ and the derivative reflectance at (a) 675 and (b) $760 \mathrm{~nm}$ in stressed leaves.

The relationship of $\rho^{\prime}$ at 675 and $760 \mathrm{~nm}$ versus $F_{v} / F_{m}$ was presented in Fig. 5, respectively, and it is observed that the derivative of red reflectance was positively correlated with $F_{v} / F_{m}$ [Fig. 5(a)] while the relationship of derivative of NIR reflectance and $F_{v} / F_{m}$ [Fig. 5(b)] was negative.

To explore whether the VIs can be applied for $F_{v} / F_{m}$ estimations under stress conditions, the samples of the experiment II that showed the obvious drop in $F_{v} / F_{m}$ (usually $5 \mathrm{~h}$ after leaf removal with $F_{v} / F_{m}$ below 0.75) were selected as stressed leaves. Due to the limitation of the spectral resolution of current sensors, it may be quite difficult to get hyperspectral data, thus, calculate $\rho^{\prime}$. Therefore, the indices in Table 1 were also tested in selected stressed leaves. Green chlorophyll index $\left(\mathrm{CI}_{\text {green }}\right), \mathrm{NDRE}_{705}$, and $\mathrm{CI}_{705}$, working well in nonstressed leaves $\left(R^{2}\right.$ above 0.64), showed very low correlations to $F_{v} / F_{m}$ in stressed leaves with $R^{2}$ below 0.28 . Normalized difference vegetation index (NDVI), MERIS terrestrial chlorophyll index (MTCI), enhanced vegetation index 2 (EVI2), and simple ratio (SR), which used red band around $680 \mathrm{~nm}$, showed significant relationships with $F_{v} / F_{m}$ in stressed leaves, but $R^{2}$ of these relationships in stressed leaves was much lower than in nonstressed leaves (Tables 2 and 3). $R^{2}$ of the relationship PRI versus $F_{v} / F_{m}$ increased significantly in stressed leaves ( 0.32 versus 0.68$)$. Many studies showed that in ecosystems when chlorophyll content changed over a wide range, PRI was highly correlated to chlorophyll variations at both leaf and canopy level, ${ }^{65,66}$ and it may be not effective for detecting vegetation photosynthetic efficiency. But PRI acted as a useful index of photosynthetic efficiency in evergreen species when chlorophyll content changed slightly and its variation was quite independent to chlorophyll. ${ }^{67}$ Thus, when leaf chlorophyll

Table 3 Best-fit functions, determination coefficients $\left(R^{2}\right)$, RMSE, and NRMSE of relationships $F_{v} / F_{m}$ versus Vls in stressed leaves.

\begin{tabular}{lllll}
\hline \hline $\mathrm{VI}$ & \multicolumn{1}{c}{ Algorithm } & $R^{2}$ & RMSE & NRMSE \\
\hline $\mathrm{NDRE}_{740}$ & $F_{v} / F_{m}=-7.08 x+0.89$ & 0.88 & 0.071 & 0.114 \\
$\mathrm{Cl}_{740}$ & $F_{v} / F_{m}=-3.11 x+0.87$ & 0.88 & 0.071 & 0.115 \\
$\mathrm{PRI}$ & $F_{v} / F_{m}=13.54 x+0.36$ & 0.68 & 0.117 & 0.188 \\
$\mathrm{MTCl}$ & $F_{v} / F_{m}=-0.46 x+1.51$ & 0.59 & 0.134 & 0.216 \\
$\mathrm{NDVI}$ & $F_{v} / F_{m}=3.64 x-2.05$ & 0.58 & 0.135 & 0.218 \\
$\mathrm{SR}$ & $F_{v} / F_{m}=0.14 x-0.31$ & 0.50 & 0.147 & 0.237 \\
$\mathrm{EVI2}$ & $F_{v} / F_{m}=1.47 x-0.12$ & 0.49 & 0.148 & 0.239 \\
$\mathrm{Cl}_{705}$ & $F_{v} / F_{m}=-0.24 x+0.84$ & 0.28 & 0.205 & 0.334 \\
$\mathrm{NDRE}_{705}$ & $F_{v} / F_{m}=-1.42 x+1.09$ & 0.27 & 0.204 & 0.333 \\
$\mathrm{Cl}_{\text {green }}$ & $F_{v} / F_{m}=-0.26 x+1.18$ & 0.26 & 0.187 & 0.301 \\
\hline \hline
\end{tabular}


content did not change significantly under short-term stress, PRI appeared more useful than chlorophyll content to indicate $F_{v} / F_{m}$ variations. Among the tested VIs, $\mathrm{CI}_{740}$ and $\mathrm{NDRE}_{740}$ were the best to estimate $F_{v} / F_{m}$ with $R^{2}$ of 0.88 and NRMSE below 0.115 . They were formulated based on the reflectance difference of two bands located at longwave red edge and NIR spectrum, which may indicate $\rho^{\prime}$ in spectral region of 750 to $900 \mathrm{~nm}$, whereas PRI may indicate $\rho^{\prime}$ in green spectral region. From Fig. 3(b), it is observed that in stressed leaves, as $F_{v} / F_{m}$ decreased, the reflectance slope in NIR the spectral region increased while it decreased in the green spectral region.

\subsection{Identify Stress Occurrence in Leaves}

$\mathrm{CI}_{740}$ and $\mathrm{NDRE}_{740}$ were accurate for $F_{v} / F_{m}$ estimates in both experiments with $R^{2}$ above 0.72 (Tables 2 and 3). However, the algorithms developed in nonstressed leaves were very different from those in stressed leaves. For nonstressed leaves, $\mathrm{CI}_{740}$ and $\mathrm{NDRE}_{740}$ positively related to $F_{v} / F_{m}$, whereas they related to $F_{v} / F_{m}$ negatively in stressed leaves [Figs. 6(a) and 6(b)]. Based on best-fit functions calibrated in two experiments, the stressed and nonstressed lines were roughly defined in VI versus $F_{v} / F_{m}$ two-dimensional (2-D) space. The nonstressed line was short with relatively high $F_{v} / F_{m}$ values. Since in healthy leaves, $F_{v} / F_{m}$ was quite conservative with small variations. The stressed line was relatively long with higher $\mathrm{CI}_{740}$ or $\mathrm{NDRE}_{740}$ values. When leaf was under possible stresses, $F_{v} / F_{m}$ dramatically decreased having wider dynamic range, and leaf reflectance decreased thus increasing VI values. Figures 6(c) and 6(d) show all samples of the experiment II in VI versus $F_{v} / F_{m}$ space. Within 0 to $5 \mathrm{~h}$, sample points were mostly located near the nonstressed line defined in the space. One or two hours later (the sixth and seventh hour), points moved toward the stressed line. After $8 \mathrm{~h}$ with no water supply, points shifted to the stressed line and quite far away from the nonstressed line. Such movements of samples in VI versus $F_{v} / F_{m}$ space may give us a hint to identify short-term stress occurrence in leaves when leaf chlorophyll content remains stable while $F_{v} / F_{m}$ tends to decrease.

Our study estimated ChlF-derived $F_{v} / F_{m}$ using spectral indices for both nonstressed and stressed leaves with promising results. The tested indices were calculated using reflectance
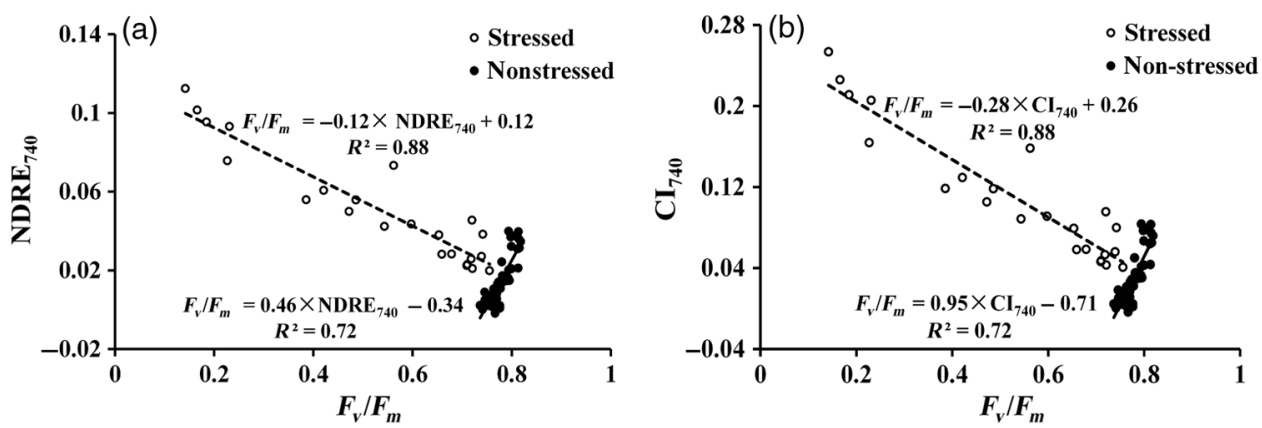

(c)
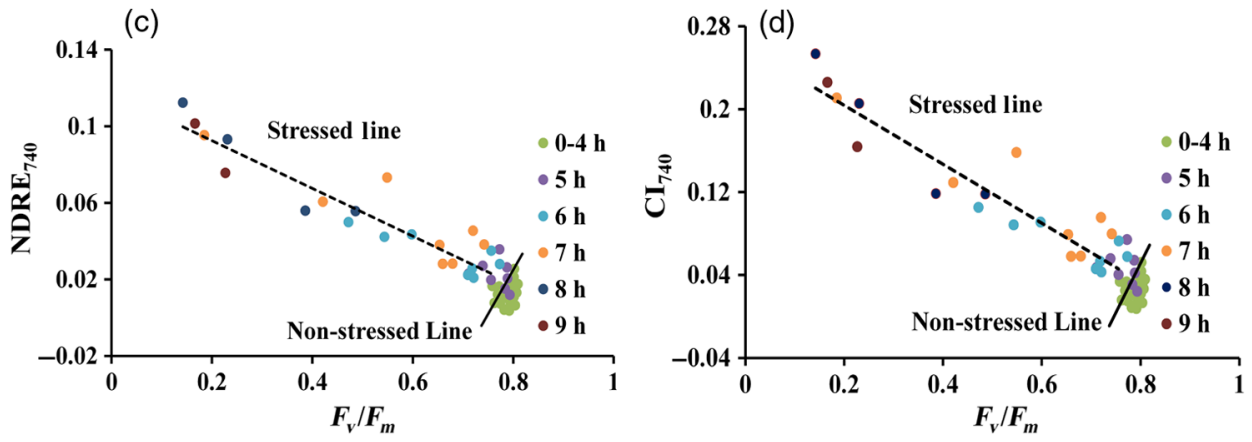

Fig. 6 Relationship of VI versus $F_{v} / F_{m}$ in stressed and nonstressed leaves for (a) $\mathrm{NDRE}_{740}$ and (b) $\mathrm{Cl}_{740}$. Sample movements within VI versus $F_{v} / F_{m} 2$-D space in leaves under short-term stress for (c) $\mathrm{NDRE}_{740}$ and (d) $\mathrm{Cl}_{740}$. 
simulated in Sentinel-2 bands, which may be easily applied to the mainstream sensors with relatively wide bands. But in this study, the experiment for stressed leaves was only conducted in lab where the light and temperature conditions might be quite different from real vegetation growing environment. Also, we studied using leaf reflectance to indicate water stress, but how the vegetation spectra will respond to other stresses (e.g., temperature and soil moisture) that may reduce photosynthesis efficiency is unclear. The next step is to apply this method for leaves in the field under various natural stresses. Furthermore, it is worthwhile to explore using reflectancederived indices to indicate ChlF information at canopy level. Vertical gradients of light and pigment distribution inside the canopy greatly increase the complexity of ChlF signals. Also, the clarification of the relationship between fluorescence and photosynthetic efficiency at canopy level is important for monitoring ChlF remotely at large scale with aircraft or satellite data.

\section{Conclusions}

We estimated a leaf ChlF-derived parameter, the intrinsic efficiency of PSII photochemistry $\left(F_{v} / F_{m}\right)$, based on leaf spectra measurements. In leaves with minimum stress, it is found that chlorophyll content closely related to $F_{v} / F_{m}$. Thus, $F_{v} / F_{m}$ can be estimated via VIs, which was developed for chlorophyll content retrieval. Several widely used indices, calculated from leaf reflectance simulated in Sentinel-2 bands, were tested to estimate $F_{v} / F_{m}$ for nonstressed leaves. All chlorophyll-related VIs worked well, and the indices using longwave red edge bands $\left(\mathrm{CI}_{740}\right.$ and $\left.\mathrm{NDRE}_{740}\right)$ were the most accurate for $F_{v} / F_{m}$ retrieval with $R^{2}$ above 0.72. For leaves under short-term stresses, the $F_{v} / F_{m}$ value began to drop but leaf chlorophyll content remained almost invariant within a day. Compared to leaf chlorophyll, the first derivative of reflectance in spectra range of 700 to $900 \mathrm{~nm}$ appeared more sensitive to $F_{v} / F_{m}$ variations. While most chlorophyll-related VIs showed low correlations with $F_{v} / F_{m}$ for leaves under stress, $\mathrm{CI}_{740}$ and $\mathrm{NDRE}_{740}$ were closely related to $F_{v} / F_{m}$ also in stressed leaves with $R^{2}$ of 0.88 . Note that $\mathrm{CI}_{740}$ or $\mathrm{NDRE}_{740}$ versus $F_{v} / F_{m}$ relationships for stressed leaves were significantly different from that for nonstressed leaves, which may give a good implication to detect short-term stress occurrence in leaves.

\section{Acknowledgments}

We acknowledge the support and the use of facilities and equipment provided by the Institute of Quantitative Remote Sensing, School of Remote Sensing and Information Engineering, Wuhan University, China. This research was supported by the National Defense Project, China (No. 30Y20A29-9003-15/17), the National 863 Project of China (No. 2013AA102401), Science Supporting Project of Hubei Province (No. 2015BCE045), and the National Natural Science Foundation of China (No. 41401390).

\section{References}

1. U. Schreiber, W. Bilger, and C. Neubauer, "Chlorophyll fluorescence as a nonintrusive indicator for rapid assessment of in vivo photosynthesis," in Ecophysiology of Photosynthesis, E. D. Schulze and M. M. Caldwell, Eds., 1st ed., pp. 49-70, Springer-Verlag, Berlin, Heidelberg (1995).

2. G. H. Krause and E. Weis, "Chlorophyll fluorescence as a tool in plant physiology: II. Interpretation of fluorescence signals," Photosynth. Res. 5, 139-157 (1984).

3. Z. Malenovsky et al., "Physically-based retrievals of Norway spruce canopy variables from very high spatial resolution hyperspectral data," in IEEE Int. Geoscience and Remote Sensing Symp. (IGARSS), Vol. 1, pp. 4057-4060 (2007).

4. A. Porcar-Castell et al., "Linking chlorophyll a fluorescence to photosynthesis for remote sensing applications: mechanisms and challenges," J. Exp. Bot. 65, 4065-4095 (2014).

5. C. V. D. Tol, W. Verhoef, and A. Rosema, "A model for chlorophyll fluorescence and photosynthesis at leaf scale," Agric. For. Meteorol. 149, 96-105 (2009). 
Peng et al.: Using remotely sensed spectral reflectance to indicate leaf photosynthetic...

6. C. Frankenberg et al., "New global observations of the terrestrial carbon cycle from GOSAT: patterns of plant fluorescence with gross primary productivity," Geophys. Res. Lett. 38, 351365 (2011).

7. Y. Zhang et al., "Estimation of vegetation photosynthetic capacity from space-based measurements of chlorophyll fluorescence for terrestrial biosphere models," Global Change Biol. 20, 3727-3742 (2014).

8. U. Schreiber, "New emitter-detector-cuvette assembly for measuring modulated chlorophyll fluorescence of highly diluted suspensions in conjunction with the standard PAM fluorometer," Z. Naturforsch. C 49, 646-656 (1994).

9. J. E. Lee, C. Frankenberg, and C. V. D. Tol, "Forest productivity and water stress in Amazonia: observations from GOSAT chlorophyll fluorescence," Proc. R. Soc. B Biol. Sci. 280, 176-188 (2013).

10. K. Maxwell and G. N. Johnson, "Chlorophyll fluorescence—a practical guide," J. Exp. Bot. 51, 659-668 (2000).

11. N. R. Baker, "Chlorophyll fluorescence: a probe of photosynthesis in vivo," Annu. Rev. Plant Biol. 59, 89-113 (2008).

12. A. Porcar-Castell, "A high-resolution portrait of the annual dynamics of photochemical and non-photochemical quenching in needles of Pinus sylvestris," Physiol. Plant. 143, 139-153 (2011).

13. I. Moya et al., "A new instrument for passive remote sensing: 1 . Measurements of sunlightinduced chlorophyll fluorescence," Remote Sens. Environ. 91, 186-197 (2004).

14. U. Rascher et al., "CEFLES2: the remote sensing component to quantify photosynthetic efficiency from the leaf to the region by measuring sun-induced fluorescence in the oxygen absorption bands," Biogeosciences 6, 1181-1198 (2009).

15. J. Joiner et al., "First observations of global and seasonal terrestrial chlorophyll fluorescence from space," Biogeosciences 8, 637-651 (2011).

16. U. Rascher, B. Gioli, and F. Miglietta, "FLEX—fluorescence explorer: a remote sensing approach to quantify spatio-temporal variations of photosynthetic efficiency from space," in Photosynthesis. Energy from the Sun, J. F. Allen and E. Gantt, Eds., pp. 1387-1390, Springer Netherlands, Berlin, Heidelberg, Germany (2007).

17. European Space Agency (ESA), "Candidate Earth Explorer Core Missions-Reports for assessment: FLEX-fluorescence explorer," ESTEC, ESA SP-1313/4, Noordwijk, The Netherlands (2008).

18. C. Frankenberg et al., "Prospects for chlorophyll fluorescence remote sensing from the Orbiting Carbon Observatory-2," Remote Sens. Environ. 147, 1-12 (2014).

19. P. J. Zarco-Tejada et al., "Steady-state chlorophyll a fluorescence detection from canopy derivative reflectance and double-peak red-edge effects," Remote Sens. Environ. 84, 283-294 (2003).

20. J. A. Plascyk, "The MK II Fraunhofer line discriminator (FLD-II) for airborne and orbital remote sensing of solar-stimulated luminescence," Opt. Eng. 14, 339-346 (1975).

21. L. Gomezchova et al., "Solar induced fluorescence measurements using a field spectroradiometer," in AIP Conf. Proc., Vol. 852, pp. 274-281 (2006).

22. L. Alonso et al., "Improved Fraunhofer line discrimination method for vegetation fluorescence quantification," IEEE Geosci. Remote Sens. 5, 620-624 (2008).

23. M. Meroni et al., "Remote sensing of solar-induced chlorophyll fluorescence: review of methods and applications," Remote Sens. Environ. 113, 2037-2051 (2009).

24. S. Z. Dobrowski et al., "Simple reflectance indices track heat and water stress-induced changes in steady-state chlorophyll fluorescence at the canopy scale," Remote Sens. Environ. 97, 403-414 (2005).

25. A. A. Gitelson, C. Buschmann, and H. K. Lichtenthaler, "The chlorophyll fluorescence ratio $\mathrm{F}_{735} / \mathrm{F}_{700}$ as an accurate measure of the chlorophyll content in plants," Remote Sens. Environ. 69, 296-302 (1999).

26. P. J. Zarco-Tejada et al., "Imaging chlorophyll fluorescence with an airborne narrow-band multispectral camera for vegetation stress detection," Remote Sens. Environ. 113, 1262 1275 (2009). 
27. M. Jia et al., "Inversion of chlorophyll fluorescence parameters on vegetation indices at leaf scale," in IEEE Int. Geoscience and Remote Sensing Symp. (IGARSS), Beijing, pp. 43594362 (2016).

28. C. Buschmann and H. K. Lichtenthaler, "Reflectance and chlorophyll fluorescence signatures of leaves," Chapter 4 in Applications of Chlorophyll Fluorescene in Photosynthesis Research, Stress Physiology, Hydrobiology and Remote Sensing, H. K. Lichtenthaler, Ed., pp. 325-332, Springer Netherlands, Berlin, Heidelberg (1988).

29. H. G. Dahn, K. P. Günther, and W. Lüdeker, "Characterisation of drought stress of maize and wheat canopies by means of spectral resolved laser induced fluorescence," EARSeL Adv. Remote Sens. 1, 12-19 (1992).

30. J. Louis, A. Ounis, and J. M. Ducruet, "Remote sensing of sunlight-induced chlorophyll fluorescence and reflectance of Scots pine in the boreal forest during spring recovery," Remote Sens. Environ. 96, 37-48 (2005).

31. G. P. Asner and A. Alencar, "Drought impacts on the Amazon forest: the remote sensing perspective," New Phytol. 187, 569-578 (2010).

32. S. Wang et al., "Monitoring and assessing the 2012 drought in the Great Plains: analyzing satellite-retrieved solar-induced chlorophyll fluorescence, drought indices, and gross primary production," Remote Sens. 8(2), 61 (2016).

33. C. Ottander and G. Oquist, "Recovery of photosynthesis in winter-stressed Scots pine," Plant Cell Environ. 14, 345-349 (1991).

34. A. Porcar-Castell et al., "Seasonal acclimation of photosystem II in Pinus sylvestris. II. Using the rate constants of sustained thermal energy dissipation and photochemistry to study the effect of the light environment," Tree Physiol. 28, 1483-1491 (2008).

35. A. I. Zygielbaum et al., "Non-destructive detection of water stress and estimation of relative water content in maize," Geophys. Res. Lett. 36, 91-100 (2009).

36. C. W. Wood et al., "Field chlorophyll measurements for evaluation of corn nitrogen status," J. Plant Nutr. 15, 487-500 (1992).

37. C. W. Wood, D. W. Reeves, and D. G. Himelrick, "Relationships between chlorophyll meter readings and leaf chlorophyll concentration, $\mathrm{N}$ status, and crop yield: a review," in Proc. of Agronomy Society New Zealand, Vol. 23, pp. 1-9 (1993).

38. Q. Ling, W. Huang, and P. Jarvis, "Use of a SPAD-502 meter to measure leaf chlorophyll concentration in Arabidopsis thaliana," Photosynth. Res. 107, 209-214 (2011).

39. R. Aroca et al., "Arbuscular mycorrhizal symbiosis influences strigolactone production under salinity and alleviates salt stress in lettuce plants," J. Plant Physiol. 170(1), 4755 (2013).

40. L. Ni et al., "Effects of artemisinin on photosystem II performance of microcystis aeruginosa by in vivo chlorophyll fluorescence," Bull. Environ. Contam. Toxicol. 89, 1165-1169 (2012).

41. M. Drusch et al., "Sentinel-2: ESA's optical high-resolution mission for GMES operational services," Remote Sens. Environ. 120, 25-36 (2012).

42. C. Atzberger and K. Richter, "Spatially constrained inversion of radiative transfer models for improved LAI mapping from future Sentinel-2 imagery," Remote Sens. Envion. 120, 208-218 (2012).

43. J. G. Clevers and A. A. Gitelson, "Remote estimation of crop and grass chlorophyll and nitrogen content using red-edge bands on Sentinel-2 and-3," Int. J. Appl. Earth Obs. Geoinf. 23, 344-351 (2013).

44. J. W. Rouse et al., "Monitoring vegetation systems in the great plains with ERTS," in Proc. of the Third Earth Resources Technology Satellite-1 Symp., NASA Special Publication 351, pp. 309-317 (1974).

45. C. F. Jordan, "Derivation of leaf-area index from quality of light on the forest floor," Ecology 50, 663-666 (1969).

46. Z. Jiang et al., "Development of a two-band enhanced vegetation index without a blue band," Remote Sens. Environ. 112, 3833-3845 (2008).

47. A. A. Gitelson, Y. Gritz, and M. N. Merzlyak, "Relationships between leaf chlorophyll content and spectral reflectance and algorithms for non-destructive chlorophyll assessment in higher plant leaves," J. Plant. Physiol. 160, 271-282 (2003). 
Peng et al.: Using remotely sensed spectral reflectance to indicate leaf photosynthetic...

48. A. A. Gitelson et al., "Remote estimation of canopy chlorophyll content in crops," Geophys. Res. Lett. 32, 93-114 (2005).

49. A. A. Gitelson and M. N. Merzlyak, "Quantitative estimation of chlorophyll-a using reflectance spectrum: experiments with autumn chestnut and maple leaves," J. Photochem. Photobiol. B 22, 247-252 (1994).

50. A. A. Gitelson and M. N. Merzlyak, "Remote estimation of chlorophyll content in higher plant leaves," Int. J. Remote Sens. 18(12), 2691-2697 (1997).

51. J. Dash and J. Curran, "The MERIS terrestrial chlorophyll index," Int. J. Remote Sens. 25, 5403-5413 (2004).

52. J. A. Gamon, J. Penuelas, and C. B. Field, "A narrow waveband spectral index that tracks diurnal changes in photosynthetic efficiency," Remote Sens. Environ. 41, 35-44 (1992).

53. C. S. T. Daughtry et al., "Estimating corn leaf chlorophyll concentration from leaf and canopy reflectance," Remote Sens. Environ. 74, 229-239 (2000).

54. D. A. Sims and J. A. Gamon, "Relationships between leaf pigment content and spectral reflectance across a wide range of species, leaf structures and developmental stages," Remote Sens. Environ. 81, 337-354 (2002).

55. F. Thenot, M. Méthy, and T. Winkel, "The photochemical reflectance index (PRI) as a waterstress index," Int. J. Remote Sens. 23, 5135-5139 (2002).

56. M. Meroni et al., "Assessing steady-state fluorescence and PRI from hyperspectral proximal sensing as early indicators of plant stress: the case of ozone exposure," Sensors 8, 1740 1754 (2008).

57. Y. Peng and A. Gitelson, "Remote estimation of gross primary productivity in soybean and maize based on total crop chlorophyll content," Remote Sens. Environ. 117, 440-448 (2012).

58. G. Aschan et al., "Photosynthetic performance of vegetative and reproductive structures of green hellebore (Helleborus viridis L. agg.)," Photosynthetica 43, 55-64 (2005).

59. Y. J. Zhang et al., "Chlorophyll fluorescence detected passively by difference reflectance spectra of wheat (Triticum aestivum L.) leaf," J. Integr. Plant Biol. 47(10) 1228-1235 (2005).

60. J. I. García-Plazaola and J. M. Becerril, "Seasonal changes in photosynthetic pigments and antioxidants in beech (Fagus sylvatica) in a Mediterranean climate: implications for tree decline diagnosis," Funct. Plant Biol. 28, 225-232 (2001).

61. R. G. Walters, "Towards an understanding of photosynthetic acclimation," J. Exp. Bot. 56, 435-447 (2005).

62. F. Yu and V. S. Berg, "Control of paraheliotropism in two phaseolus species," Plant Physiol. 106, 1567-1573 (1994).

63. M. Kasahara et al., "Chloroplast avoidance movement reduces photodamage in plants," Nature 420, 829-832 (2002).

64. P. J. Zarco-Tejada et al., "Chlorophyll fluorescence effects on vegetation apparent reflectance: I. Leaf-level measurements and model simulation," Remote Sens. Environ. 74, 582595 (2000).

65. A. A. Gitelson, J. A. Gamon, and A. Solovchenko, "Mulitiple drivers of seasonal change in PRI: implications for photosynthesis 1. Leaf level," Remote Sens. Environ. 191, 110-116 (2017).

66. A. A. Gitelson, J. A. Gamon, and A. Solovchenko, "Multiple drivers of seasonal change in PRI: implications for photosynthesis 2. Stand level," Remote Sens. Environ. 190, 198-206 (2017).

67. Y. Peng et al., "Remote estimation of gross primary production in maize and support for new paradigm based on total crop chlorophyll content," Remote Sens. Environ. 115, 978-989 (2011).

Yi Peng received her PhD from the School of Natural Resources, University of NebraskaLincoln, USA, in 2012. Currently, she is the associate professor in the School of Remote Sensing and Information Engineering, Wuhan University, China. Her research interests focus on quantitative remote sensing of agriculture. 
Peng et al.: Using remotely sensed spectral reflectance to indicate leaf photosynthetic...

Shenghui Fang is the director of the Institute of Quantitative Remote Sensing, School of Remote Sensing and Information Engineering, Wuhan University, China. His research interests include quantitative remote sensing, image processing and microwave remote sensing.

Yan Gong works as the associate director of the Institute of Quantitative Remote Sensing, School of Remote Sensing and Information Engineering, Wuhan University, China. His research interests include remote sensing of agriculture and image analysis.

Kan Liu received his PhD form the Institute of Pattern Recognition and Artificial Intelligence, Huazhong University of Science and Technology, China, in 2012. He is currently working in Wuhan Institute of Physics and Mathematics of Chinese Academy of Sciences, China. His research interests include bioinformatics, fluorescent data analysis, and integrated spectral imaging devices.

Biographies for the other authors are not available. 